\title{
Combining Lactate Dehydrogenase and Fibrinogen: Potential Factors to Predict Therapeutic Efficacy and Prognosis of Patients with Small-Cell Lung Cancer
}

\author{
Wen Huang ${ }^{1} * *$ \\ Ping Liu (iD ${ }^{\prime} * *$ \\ Min Zong ${ }^{2, *}$ \\ Qian-Qian Chen (D) \\ Hong Zhou' \\ Hui Kong ' \\ Wei-Ping Xie' \\ 'Department of Respiratory and Critical \\ Care Medicine, The First Affiliated \\ Hospital of Nanjing Medical University, \\ Nanjing, 210029, People's Republic of \\ China; ${ }^{2}$ Department of Radiology, The \\ First Affiliated Hospital of Nanjing \\ Medical University, Nanjing, 210029, \\ People's Republic of China \\ *These authors contributed equally to \\ this work
}

Background: A growing interest exists in identifying reliable and low-cost biomarkers or factors that could predict the therapeutic response, prognosis, recurrence, and survival in smallcell lung cancer (SCLC). This study aimed to investigate the better predictors of chemotherapy efficacy and prognosis in patients with SCLC receiving first-line chemotherapy and radiotherapy. Materials and Methods: This study retrospectively retrieved the medical records of patients with SCLC treated with first-line platinum-based chemotherapy and radiotherapy from January 2016 to June 2019 in the First Affiliated Hospital of Nanjing Medical University. Plasma biochemical parameters, clinical features, and overall survival (OS) time were collected. The independent effects of plasma parameters on patient survival were assessed by conducting univariate and multivariate Cox regression analyses. The optimal cut-off values of independent risk factors in the ROC curve and Kaplan-Meier survival analysis were determined using MedCalc software.

Results: Statistically significant differences in lactate dehydrogenase (LDH) and fibrinogen (Fbg) were found between the complete remission + partial remission group and the nonresponders, which consisted of stable-disease and progressive-disease groups, after first-line chemotherapy. Multivariate Cox regression analysis showed that LDH and Fbg were independent risk factors in predicting PFS (LDH HR: 1.013, 95\% CI: $1.002-1.030, P=0.037$; Fbg HR: $1.622,95 \% \mathrm{CI}: 1.094-2.526, P=0.017)$ and OS (LDH HR: $1.021,95 \% \mathrm{CI}$ : 1.008-1.034, $P=0.001$; Fbg HR: 2.168, 95\% CI: $1.324-3.550, P=0.002)$. The AUC of $\mathrm{LDH}$ and Fbg was 0.77 and 0.745 , respectively. The cut-off value of LDH and Fbg in predicting OS was $263 \mathrm{U} / \mathrm{L}$ and $4.03 \mathrm{~g} / \mathrm{L}$. When these two data were combined, the AUC reached 0.832 , better than that of LDH and Fbg alone. The objective response rate (ORR) and OS were significantly different among these three different groups according to the addition of the assigned value $(P<0.05)$.

Conclusion: Combined retreatment serum LDH and Fbg levels may be a better potential biomarker for predicting the clinical efficacy of chemotherapy and the prognosis of individuals with SCLC. Combining these two parameters could improve prediction efficacy.

Keywords: lactate dehydrogenase, fibrinogen, overall survival, prognosis, small cell lung cancer

\section{Introduction}

The mortality rates of lung cancer, the most common type of cancer, rank the highest among all cancer-related deaths around the world. ${ }^{1}$ Small-cell lung cancer (SCLC) comprises 13-20\% of lung cancer. ${ }^{2,3}$ Although the median overall survival (OS) of SCLC rarely reaches more than 1 year, the prognosis of patients in the same 
stage of SCLC still varies. As a result, a growing interest exists in identifying reliable and low-cost biomarkers or factors that could predict the therapeutic response, prognosis, recurrence, and survival in SCLC. Several biomarkers have been identified as being related to the prognosis of SCLC, but due to various reasons, their clinical applications have been limited. Therefore, the identification of effective and easily available biomarkers for the prognosis of SCLC is of clinical significance.

Serum lactate dehydrogenase (LDH) has been proven to be related to metabolism in tumors, and it has been used for detecting malignant tumors in recent years. LDH concentration is a prognostic marker in many kinds of tumors. $^{4-8}$ Petrelli et al $^{9}$ found that the LDH level before treatment in patients with lung cancer was inversely correlated with worsened OS. As to SCLC, Zhang et al conducted a systematic review with meta-analysis and suggested a significant correlation between elevated serum LDH levels and poor OS in patients with SCLC. ${ }^{10}$

Inflammation is essential for cancer development and invasion because it enhances angiogenesis, accelerates cancer cell proliferation, and promotes tumor metastasis. Mounting evidence demonstrated that systemic inflammatory response in carcinomas is a significant predictor for the outcome. ${ }^{11-13}$ Cancer-related inflammation is composed of different inflammatory cells and factors or chemokines. Neutrophil-to-lymphocyte ratio (NLR) and platelet-to-lymphocyte ratio (PLR) were associated with prognosis in various cancers, including SCLC. ${ }^{13-16}$ The interleukin (IL)-6 released by tumor cells or immunocytes in the cancer microenvironment could increase the concentration of acute-phase reaction proteins, including C-reactive protein and fibrinogen (Fbg), in liver. ${ }^{13-15}$ Fan et $\mathrm{al}^{16}$ found that the Fbg level in serum was inversely correlated with SCLC prognosis in a group of 120 patients with SCLC. Zhu et al found that after two cycles of chemotherapy, patients with SCLC who had higher levels of Fbg and D-dimer exhibited poor reactions to chemotherapy and low survival rate. ${ }^{17}$ However, which among the above biomarkers reported as useful for predicting the therapeutic effect and survival in patients with SCLC are better than the others in clinical use have not been studied before.

The present study aimed to explore the prognostic effect of LDH, albumin (Alb), whole blood count, D-Dimer, Fbg, and neuron-specific enolase (NSE) in patients with SCLC after being treated via first-line platinum-based chemotherapy and radiotherapy and achieve better prognostic predictors.

\section{Materials and Methods}

\section{Patients}

This study was approved by the ethics committee of the First Affiliated Hospital of Nanjing Medical University (Nanjing, China). Informed consent was waived since this was a retrospective study and the study protocol conformed to the ethical guidelines of the Declaration of Helsinki. All patient data accessed complied with relevant data protection and privacy regulations. The records of 220 patients with SCLC in our institution from January 2016 to June 2019 were reviewed. The inclusion criteria were as follows: (1) cytological or histological diagnosis of SCLC, (2) pretreatment hematological results obtained before any anti-cancer treatment, (3) Eastern Cooperative Oncology Group grade rank of 0-2, (4) availability of complete patient records, and (5) first-line chemotherapy of etoposide and cisplatin. The exclusion criteria were as follows: (1) patients without complete clinical and pathological data, (2) patients with other malignancies, (3) patients who received regular anticoagulation therapy, and (4) patients who did not receive radiotherapy. Finally, the records of 161 SCLC patients were included.

\section{Data Collection}

Baseline characteristics such as gender, age, clinical or postoperative stage, smoking status, and hematological results were retrieved using an electronic medical record system. The hematological results were obtained within 10 days before the first treatment. The acquired results included the measurement of $\mathrm{LDH}$, Alb, whole blood count, D-Dimer, Fbg, and NSE levels. All data were anonymized to ensure privacy. Therapeutic response was evaluated in accordance with the Response Evaluation Criteria in Solid Tumors (version 1.1), including complete remission (CR) and partial remission (PR), while nonresponders consisted of stable disease (SD) and progressive disease (PD). The progression-free survival (PFS) and OS information was obtained via regular monitoring and defined as a dichotomous variable $(1=$ dead; $0=$ alive $)$.

\section{Follow-Up}

After the first-line therapy, all the patients were followed up to obtain the survival data by means of medical record 
retrieval or telephone interview, and the last follow-up was on December 31, 2019. The dependent variable OS was calculated from the date of SCLC diagnosis to death or the last follow-up. One-year OS was the determined endpoint in this study.

\section{Statistical Analysis}

Continuous data were expressed as mean or median \pm standard deviation. One-way ANOVA was used to analyze the associations between therapeutic response and clinical data. The Cox proportional hazard regression model was used to assess the predictive value of $\mathrm{LDH}$, Alb, whole blood count, D-Dimer, Fbg, and NSE levels for OS. The ROC curve, Kaplan-Meier survival curve, and the optimal cut-off values of each candidate hematological biomarker for survival prediction were calculated using MedCalc software. Approximation of value assignment was used to combine the Fbg and $\mathrm{LDH}$ according to the cut-off value of $\mathrm{Fbg}$ and $\mathrm{LDH}$. According to the addition of the assigned value, three groups were assigned. Log rank test was used for survival comparisons between different groups. All statistical tests were performed on SPSS version 19.0 (IBM, Armonk, NY, USA) and MedCalc. $P$ values $<0.05$ were considered statistically significant.

\section{Results}

\section{Patient Characteristics and Treatments}

A total of 161 patients with SCLC were included in this study. Their baseline characteristics are shown in Table 1. Ninety-one cases were diagnosed as limited disease (LD)stage SCLC, while the rest were confirmed as extensive disease (ED)-stage SCLC. The average age of onset was 62 years (range, 25-91 years). Smoking history was noted in $89.4 \%$ of the patients. The first-line chemotherapy regimen was etoposide combined with cisplatin. A total of 91 patients received radiotherapy after chemotherapy, while 70 received concurrent chemo-radiotherapy. Fifty-six patients were evaluated as CR (10) and PR (46) after 4-6 cycles of chemotherapy. The serum median or mean concentrations of LDH, Alb, whole blood count, D-Dimer, Fbg, and NSE levels were calculated. After four cycles of first-line chemotherapy, 56 patients achieved objective response rate $\mathrm{ORR},(\mathrm{CR}+\mathrm{PR}=34.8 \%)$. Among the remaining patients, 63 achieved SD (63) and the rest progressed (42), in which $79(49.1 \%)$ patients were at LD stage and $82(50.9 \%)$ were at ED stage.
Table I Clinical and Pathological Characteristics of I6I Eligible Patients with Lung Cancer

\begin{tabular}{|c|c|}
\hline Characteristics & Value (Average \pm SD) \\
\hline Total & 161 \\
\hline Age (years) & $62.98 \pm 9.68$ \\
\hline \multicolumn{2}{|l|}{ Sex } \\
\hline Male & 140 \\
\hline Female & 21 \\
\hline \multicolumn{2}{|l|}{ Smoking status } \\
\hline Smoker & $|4|$ \\
\hline Never smoked & 20 \\
\hline NSE (ng/mL) & $57.41 \pm 58.56$ \\
\hline LDH (U/L) & $247.19 \pm 99.66$ \\
\hline Fbg $(g / L)$ & $4.087 \pm 1.48$ \\
\hline Albumin $(g / L)$ & $38.80 \pm 4.60$ \\
\hline D-D (mg/L) & $0.62 \pm 0.81$ \\
\hline NLR & $3.08 \pm 1.64$ \\
\hline PLR & $162.78 \pm 82.78$ \\
\hline \multicolumn{2}{|l|}{ Stage } \\
\hline Limited stage & 79 \\
\hline Extensive stage & 82 \\
\hline \multicolumn{2}{|l|}{ Tumor response } \\
\hline$C R+P R$ & 56 \\
\hline$S D+P D$ & 105 \\
\hline
\end{tabular}

Note: Data were presented as mean/median \pm standard deviation (SD).

Abbreviations: ED, extensive disease; LD, limited disease; NLR, neutrophi//lymphocyte ratio; PLR, platelet/lymphocyte ratio; CR, complete response; $L D H$, lactate dehydrogenase; NSE, neuron-specific enolase; PD, progress disease; PR, partial response; SD, stable disease; Fbg, fibrinogen.

\section{Correlation of Stage, NSE, Alb, NLR, PLR,} D-Dimer, Fbg, and LDH Levels with ORR

Patients with CR and PR showed lower NSE levels [41.99 (11.27-118.7) $\mathrm{ng} / \mathrm{mL}$ ] than those who achieved SD and PD [50.82 (9.01-330) and $71.4(14.36-370) \mathrm{ng} / \mathrm{mL}$ respectively, $P=0.030]$ (Table 2). The pretreatment LDH levels of patients who achieved $\mathrm{CR}+\mathrm{PR}$ were significantly lower than those of patients who achieved SD and PD [207.9 (65-380) IU/L vs 240 (139-752) and 306.44 (144-664) IU/L, $P<0.001]$. The pretreatment Fbg levels in patients who achieved CR + PR were significantly lower than those in patients who achieved SD and PD [3.7 (1.92-7.05) IU/L vs 3.81 (1.22-8.02) and 4.95 
Table 2 Correlation of NSE, Albumin, NLR, PLR, D-Dimer, Fibrinogen, and LDH Levels with Clinical Efficacy (ORR)

\begin{tabular}{|l|l|l|l|l|}
\hline & CR + PR (n = 56) & SD (n= 63) & PD (n = 42) & P-value \\
\hline Age & $64.63 \pm 10.40$ & $62.27 \pm 9.38$ & $61.83 \pm 9.30$ & 0.41 I \\
\hline Sex (male, \%) & 82.50 & 86.36 & 96.66 & 0.085 \\
\hline Smoking (No-smoked, \%) & 29.79 & 24.00 & 13.89 & 0.217 \\
\hline LDH (U/L) & $207.90 \pm 62.43$ & $240.00 \pm 104.68$ & $306.44 \pm 116.26$ & $<0.001 *$ \\
\hline Fibrinogen (g/L) & $3.70 \pm 1.21$ & $3.81 \pm 1.50$ & $4.95 \pm 1.45$ & 0.002 \\
\hline NSE (ng/mL) & $41.99 \pm 25.25$ & $50.82 \pm 559.78$ & $71.4 \pm 72.32$ & $0.030^{*}$ \\
\hline Albumin(g/L) & $39.75 \pm 3.17$ & $37.6 \pm 4.72$ & $35 \pm 5.71$ & $0.039 *$ \\
\hline D-D (mg/L) & $0.35 \pm 0.36$ & $0.3 \pm 0.68$ & $0.44 \pm 1.33$ & 0.175 \\
\hline NLR & $2.77 \pm 1.42$ & $2.82 \pm 1.13$ & $3.13 \pm 2.42$ & $0.049 *$ \\
\hline PLR & $139.18 \pm 64.65$ & $127.46 \pm 78.7$ & $149.59 \pm 116.6$ & 0.357 \\
\hline Stage & 27 & 34 & 18 & 0.618 \\
\hline
\end{tabular}

Notes: Data were presented as median with standard deviation (SD). Difference among three groups was determined using Kruskal-Wallis $\mathrm{H}$ rank sum test. $* P$ values $<$ 0.05 were considered significant.

(2.32-7.05) IU/L, $P=0.002]$, while decreased Alb levels showed unfavorable chemotherapy results (SD and PD) [39.75 (34.8-45.6) IU/L vs $37.64(28-49)$ IU/L and 35 (24.8-46.3) IU/L, $P=0.039]$. However, no significant difference in the D-Dimer level was found in patients with different therapeutic responses $(P=0.175)$.

\section{Associations of Stage, NSE, Alb, NLR, PLR, D-Dimer, Fbg, and LDH with PFS}

As shown in Table 3, stage, serum NSE, Alb, PLR, D-Dimer, Fbg, and LDH were significantly associated with PFS (Stage HR: 1.182, 95\% CI: 1.002-2.641, $P=$ 0.001 ; NSE HR: 1.024 , $95 \%$ CI: $1.010-1.038$, $\mathrm{P}=0.001$; LDH HR: 1.012, 95\% CI: 1.005-1.019, $\mathrm{P}=$ 0.001; Alb HR: $0.891,95 \%$ CI: 0.815-0.976, $\mathrm{P}=0.012$; D-Dimer HR: 4.177, 95\% CI: 14.79-11.795, $\mathrm{P}=0.007$; Fbg HR: 2.304, 95\% CI: 1.539-3.449, P < 0.001; PLR HR: 1.007, 95\% CI: 1.001-1.014, $\mathrm{P}=0.018)$. Multivariate analysis showed that LDH (HR: 1.013, 95\% CI: 1.002-1.030, $\mathrm{P}=0.037$ ), and Fbg (HR: 1.622, 95\% CI: $1.094-2.526, \mathrm{P}=0.017$ ) were significantly associated with PFS.

Table 3 Univariate and Multivariate Cox Models for Progression-Free Survival

\begin{tabular}{|c|c|c|c|c|c|c|}
\hline \multirow[t]{2}{*}{ Variables } & \multicolumn{3}{|c|}{ Univariate Cox Regression } & \multicolumn{3}{|c|}{ Multivariate Cox Regression } \\
\hline & HR & $95 \% \mathrm{Cl}$ & $\boldsymbol{P}$ & HR & $95 \% \mathrm{Cl}$ & $P$ \\
\hline Stage & 1.182 & $1.002-2.64 \mid$ & $0.001 *$ & 0.615 & $0.211-1.797$ & 0.375 \\
\hline Age & 0.989 & $0.951-1.028$ & 0.565 & - & - & - \\
\hline Sex & 0.813 & $0.254-2.602$ & 0.727 & - & - & - \\
\hline Smoking & I & $0.999-1.001$ & 0.757 & - & - & - \\
\hline CEA & 1.025 & $0.999-1.05 \mid$ & 0.062 & - & - & - \\
\hline NSE & 1.024 & $1.010-1.038$ & $0.00 I^{*}$ & 1.010 & $0.999-1.021$ & 0.079 \\
\hline $\mathrm{LDH}$ & 1.012 & $1.005-1.019$ & $0.001 *$ & 1.013 & $1.002-1.030$ & $0.037^{*}$ \\
\hline Albumin & 0.891 & $0.815-0.976$ & $0.012^{*}$ & 1.005 & $0.895-1.128$ & 0.938 \\
\hline D-D & 4.177 & $|479-| \mid .795$ & $0.007^{*}$ & 1.038 & $0.474-2.276$ & 0.925 \\
\hline Fibrinogen & 2.304 & I.539-3.449 & $<0.00 I^{*}$ & 1.622 & $1.094-2.526$ & $0.017 *$ \\
\hline NLR & 1.270 & $0.976-1.653$ & 0.075 & - & - & - \\
\hline PLR & 1.007 & $1.001-1.014$ & $0.018^{*}$ & 0.998 & $0.991-1.006$ & 0.686 \\
\hline
\end{tabular}

Note: $* P$ values $<0.05$ were considered significant. 
Table 4 Univariate and Multivariate Cox Models for Overall Survival

\begin{tabular}{|c|c|c|c|c|c|c|}
\hline \multirow[t]{2}{*}{ Variables } & \multicolumn{3}{|c|}{ Univariate Cox Regression } & \multicolumn{3}{|c|}{ Multivariate Cox Regression } \\
\hline & HR & $95 \% \mathrm{Cl}$ & $P$ & HR & $95 \% \mathrm{Cl}$ & $P$ \\
\hline Stage & 2.353 & $1.094-5.062$ & $0.029 *$ & 1.364 & $0.42 I-4.412$ & 0.605 \\
\hline CEA & 1.032 & $1.004-1.061$ & $0.024^{*}$ & 1.019 & $0.979-1.061$ & 0.362 \\
\hline NSE & 1.009 & $1.005-1.012$ & $<0.00 I^{*}$ & 1.002 & $0.987-1.017$ & 0.784 \\
\hline $\mathrm{LDH}$ & 1.018 & $1.010-1.027$ & $<0.00 I^{*}$ & 1.021 & $1.008-1.034$ & $0.001 *$ \\
\hline Albumin & 0.872 & $0.872-0.936$ & $<0.00 I^{*}$ & 0.794 & $0.676-0.933$ & $0.005^{*}$ \\
\hline D-D & 1.507 & $1.314-2.004$ & $0.005^{*}$ & 0.317 & $0.104-0.964$ & $0.043^{*}$ \\
\hline Fibrinogen & 1.742 & $1.393-2.179$ & $<0.00 I^{*}$ & 2.168 & $1.324-3.550$ & $0.002^{*}$ \\
\hline NLR & 1.234 & I.053-I.447 & $0.010^{*}$ & 1.052 & $0.634-1.744$ & 0.846 \\
\hline PLR & 1.005 & $1.002-1.008$ & $0.002^{*}$ & 1.002 & $0.990-1.014$ & 0.727 \\
\hline
\end{tabular}

Note: ${ }^{*} P$ values $<0.05$ were considered statistically significant.

\section{Associations of Stage, NSE, Alb, NLR, PLR, D-Dimer, Fbg, and LDH with OS}

As shown in Table 4, univariate analysis showed that the following variables were significantly associated with OS: stage, CEA, NSE, D-Dimer, Alb, LDH, PLR, NLR, and Fbg (Stage HR: 2.353, 95\% CI: 1.094-5.062, $P=0.029$; CEA HR: 1.032, 95\% CI: $1.004-1.061, P=0.024$; NSE HR: $1.009,95 \%$ CI: $1.005-1.012, P<0.001$; LDH HR: $1.018,95 \%$ CI: $1 .-$ 010-1.027, $P<0.001$; Alb HR: 0.872, 95\% CI: 0.870-0.936, $P<0.001$; D-Dimer HR: 1.507, 95\% CI: 1.314-2.004, $P=$ 0.005; Fbg HR: 1.742, 95\% CI: 1.393-2.179, $P<0.001$; NLR HR: 1.234, 95\% CI: 1.053-1.447, $P=0.010$; PLR HR: 1.005, 95\% CI: $1.002-1.008, P=0.002)$. Afterwards, all factors with $P$ value $<0.05$ were analyzed via multivariate Cox hazard regression analysis, and the results showed that the following variables were significantly associated with OS: Alb (albu$\mathrm{min}$ ), LDH, Fbg and D-Dimer (Alb HR: 0.794, 0.676-0.933, $P<0.001$; LDH HR: $1.021,95 \%$ CI: $1.008-1.034, P=0.001$; Fbg HR: 2.168, 95\% CI: 1.324-3.550, $P=0.002$; D-Dimer HR: $0.317,0.104-0.964, P<0.001)$.

\section{Effects of LDH and Fbg Alone on Prognostic Abilities}

The AUC values of LDH and Fbg were 0.77 (95\% CI: 0.651 to 0.825 ) and 0.745 , respectively, while optimal thresholds for survival prediction in patients with SCLC were $263 \mathrm{U} / \mathrm{L}$ (Sensitivity $56.52 \quad$ (95\% CI: 41.1-71.1\%), Specificity 89.86\% (95\% CI: 80.2-95.8\%)) and $4.03 \mathrm{~g} / \mathrm{L}$ (Sensitivity 68.29\% (95\% CI: 51.9-81.9\%), Specificity 73.44\% (95\% CI: 60.9-83.7\%)), respectively (Figure 1). Subsequently, in accordance with the best cut-off values of these biomarkers, patients were further divided into low or high (defined by ROC curve, cut-off values of 263 (U/L), and $4.03 \mathrm{~g} / \mathrm{L}$ groups). Significant differences in 1-year OS were observed between the patients with low and high LDH $(P<0.001$; Figure 2A) and low and high $\mathrm{Fbg}(P<0.001$; Figure 2B).

\section{Effects of Combining LDH and Fbg on ORR and Prognostic Abilities}

The AUC when LDH and Fbg were combined was 0.83 (95\% CI: 0.746 to 0.898 ), optimal thresholds for
A

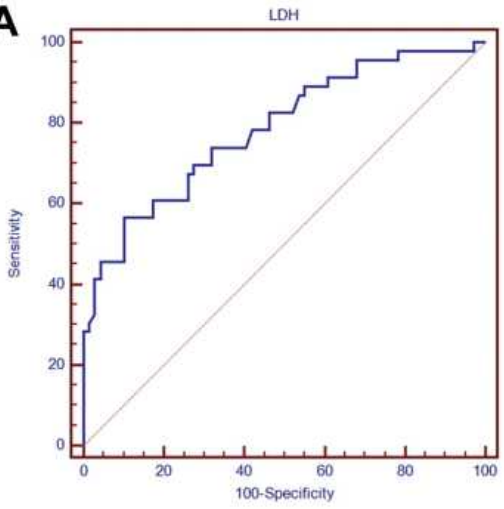

B

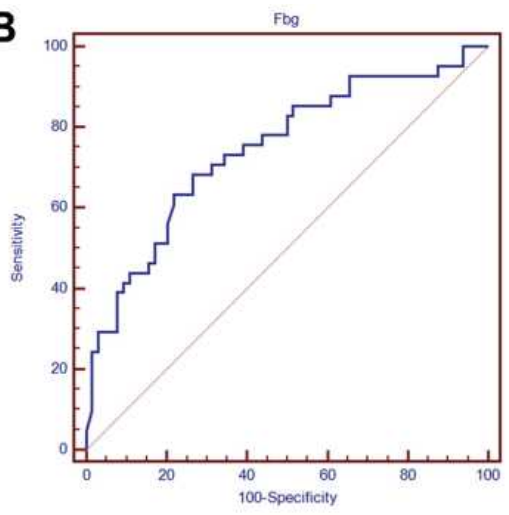

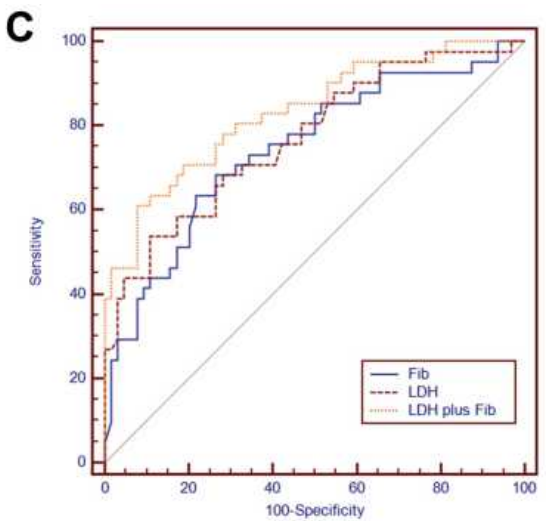

Figure I Receiver-operating characteristic curves for (A) LDH, (B) Fbg, and (C) comparison among LDH, Fbg, and combined LDH and Fbg. 

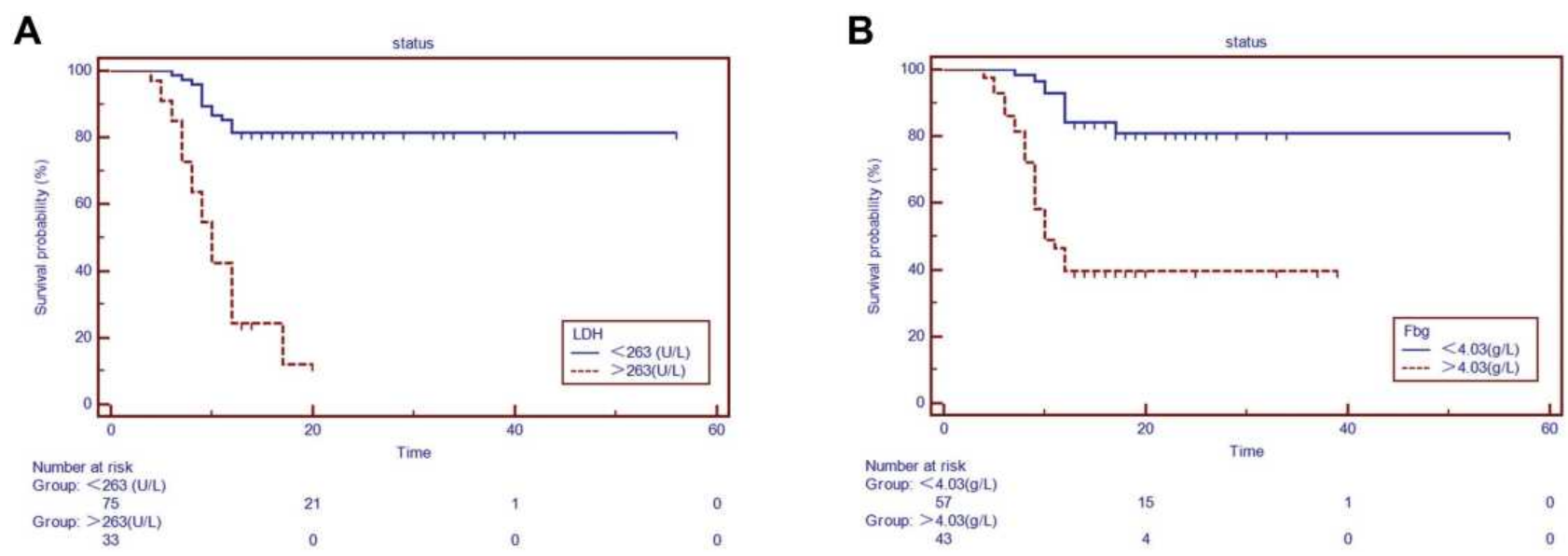

Figure 2 Kaplan-Meier survival curves of overall survival according to LDH and Fbg in SCLC. (A) (-) LDH $<263$ (U/L) and (-) LDH $>263 \mathrm{U} / \mathrm{L}$. (B) (-) Fbg $<4.03 \mathrm{~g} / \mathrm{L}$ and (-) $\mathrm{Fbg}>4.03 \mathrm{~g} / \mathrm{L}$.

LDH plus Fbg

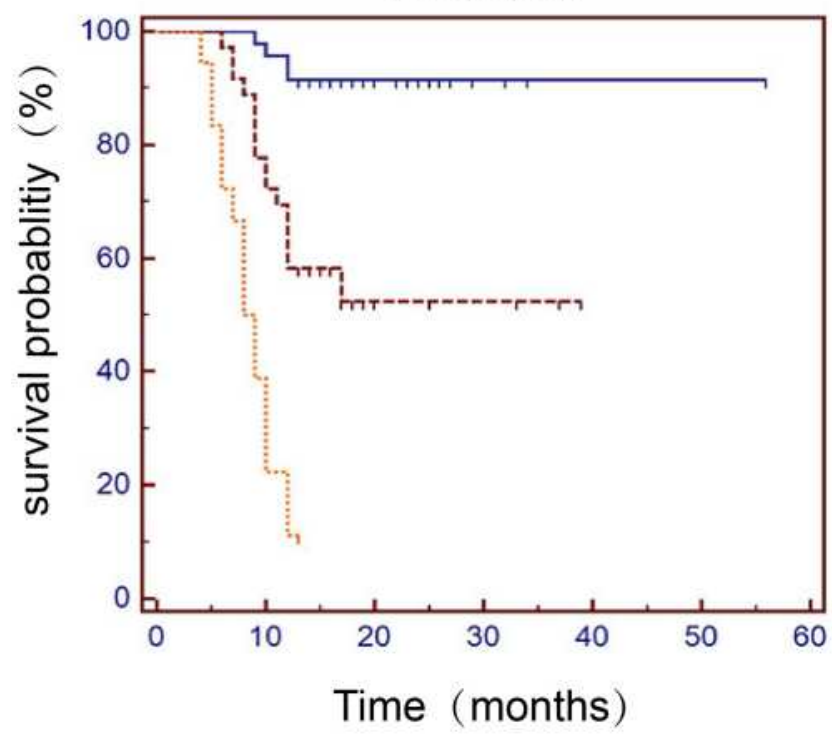

$\mathrm{LDH}<263(\mathrm{U} / \mathrm{L}), \mathrm{Fbg}<4.03 \mathrm{~g} / \mathrm{L}$

$\mathrm{Fbg}>4.03 \mathrm{~g} / \mathrm{L}, \mathrm{LDH}<263(\mathrm{U} / \mathrm{L}) ; \mathrm{LDH}>263(\mathrm{U} / \mathrm{L}), \mathrm{Fbg}<4.03 \mathrm{~g} / \mathrm{L}$ $\mathrm{LDH}>263(\mathrm{U} / \mathrm{L}), \mathrm{Fbg}>4.03 \mathrm{~g} / \mathrm{L}$

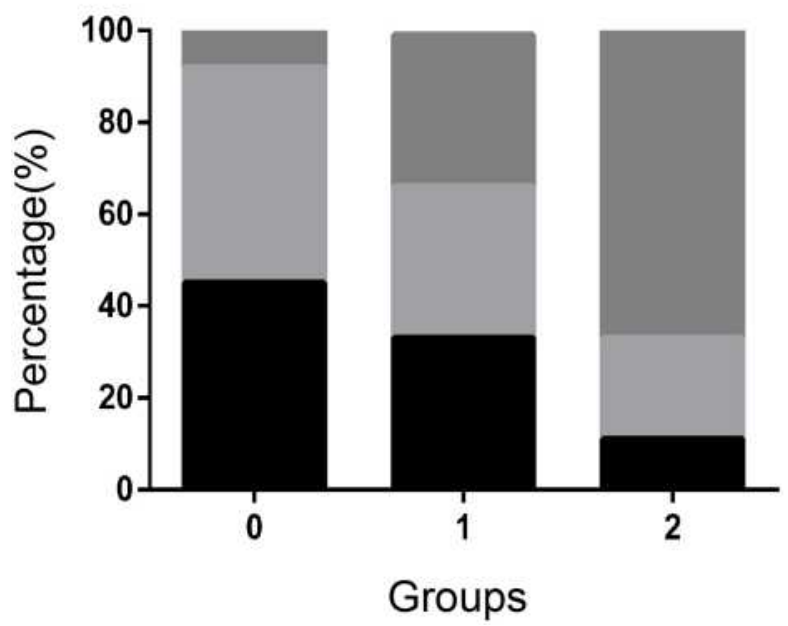

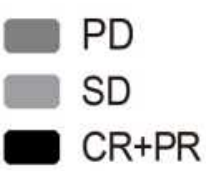

$0: \mathrm{LDH}<263(\mathrm{U} / \mathrm{L}), \mathrm{Fbg}<4.03 \mathrm{~g} / \mathrm{L}$

1: Fbg $>4.03 \mathrm{~g} / \mathrm{L}, \mathrm{LDH}<263(\mathrm{U} / \mathrm{L}) ; \mathrm{LDH}>263(\mathrm{U} / \mathrm{L}), \mathrm{Fbg}<4.03 \mathrm{~g} / \mathrm{L}$

2: $\mathrm{LDH}>263(\mathrm{U} / \mathrm{L}), \mathrm{Fbg}>4.03 \mathrm{~g} / \mathrm{L}$

Figure 3 Kaplan-Meier survival curves of overall survival and ORR according to combining LDH and Fbg in SCLC. 
survival prediction was 0.5538 ((Figure 1, sensitivity 60.98\% (95\% CI: 44.5-75.8\%), Specificity 92.19\% (95\% CI: 82.7-97.4\%)). Subsequently, in accordance with the best cut-off values of LDH $(263(\mathrm{U} / \mathrm{L}))$ and Fbg $(4.03 \mathrm{~g} / \mathrm{L})$, patients were further divided into low, medium, and high (defined by approximation of value assignment, that is Fbg $>4.03 \mathrm{~g} / \mathrm{L}$ was assigned $1, \mathrm{Fbg}$ $<4.03 \mathrm{~g} / \mathrm{L}$ was assigned 0, meanwhile, $\mathrm{LDH}>263$ (U/ L) was assigned $1, \mathrm{LDH}<263(\mathrm{U} / \mathrm{L})$ was assigned 0 . And we group the patients per this combination according to the addition of the assigned value, that is $0,1,2$. Significant differences in 1-year OS and ORR were observed between the patients of the low, medium, and high groups $(P<0.001$; Figure 3$)$.

\section{Discussion}

This study revealed that LDH and Fbg were significant and independent predictive factors for the prognosis of SCLC. In patients who achieved CR + PR, lower levels of LDH and Fbg were observed with longer PFS and OS after first-line chemotherapy and radiotherapy. In addition, the AUC of the combination of LDH and Fbg was better than that of LDH and Fbg alone.

Fbg is an acute-phase reactant protein and a plasma coagulation factor that plays an important role in increasing plasma viscosity, platelet aggregation, facilitating fibrin deposition, vasoconstriction, and promoting growth factor release. It is synthesized by hepatocytes, and it plays a proinflammatory role in certain conditions. ${ }^{20}$ It is involved in fibrinolysis, wound healing, inflammatory responses, and oncogenesis. ${ }^{21}$ Increased plasma Fbg levels reflect aberrantly activated coagulation and fibrinolysis. Buccheri et al and Lillicrap et al indicated that patients with lung cancer patients have an increased tendency of blood coagulation and abnormal fibrinolytic system. ${ }^{22,23}$ Statistically significant differences in circulating inflammatory proteins, such as Fbg and Alb, were commonly observed in patients with lung cancer compared with healthy individuals. ${ }^{24}$ Previous studies indicated that increased Fbg levels were correlated with distant tumor metastasis and poor outcomes in different kinds of cancer. ${ }^{18,19}$ Fan et $\mathrm{al}^{16}$ found that elevated plasma Fbg was independently associated with prognosis in patients with SCLC. In the present study, the Fbg level was associated with therapeutic efficiency and survival in SCLC. Moreover, in the past decades, several studies have demonstrated that inflammation has an important role in lung cancer development and progression. A vicious circle between inflammation and cancer is that chronic inflammation promotes oncogenesis, while tumor cells recruit inflammatory cells to promote further growth and ultimately metastasis. In animal models, Fbg could increase the metastatic potential. ${ }^{25}$ Tumor cell invasion and micrometastasis were suppressed in Fbg-deficient mice, indicating that $\mathrm{Fbg}$ is one of the determinants of spontaneous metastatic potential. ${ }^{26}$ These findings supported that Fbg could be a predictor for the prognosis of SCLC.

$\mathrm{LDH}$ is a tetrameric enzyme that could catalyze the redox reaction between lactic acid and pyruvic acid. ${ }^{29}$ In the tumor environment, an anaerobic condition that catalyses the redox reaction between lactic acid and pyruvic acid is usually observed. High LDH levels are a reflection of anaerobic glycolytic metabolism in intra-tumoral environment. ${ }^{30}$ A total of 139 patients with SCLC were retrospectively included in the study of Liu et $\mathrm{al}^{31}{ }^{31}$ who identified that high NLR $(>4.55)$ and PLR $(>148)$ levels were not only proportionally related to elevated LDH and low Alb levels but also a predictor of mortality. An increase in pretreatment LDH concentration in patients with lung cancer was associated with worsened OS. The result of the present study is in accordance with that of previous reports. Given that serum LDH level increased with tumor progression, it was identified as a prognostic marker for cancer. Hermes et $\mathrm{al}^{8}$ found that increased LDH levels were an independent factor to predict mortality and morbidity in patients with SCLC receiving chemotherapy. The LDH level in serum was also confirmed as a predictor for chemotherapy response and prognosis in patients with SCLC treated. ${ }^{10,27,28}$ These reports were broadly in line with the current study, that is, increased concentrations of serum LDH were related to unsatisfactory chemotherapy response and poor survival of patients with SCLC. The above results all indicated that LDH is an effective biomarker for the prognosis of SCLC.

No significant differences were found among NSE, PLR, NLR, D-D, and Alb in this study. Hirose et al enrolled 103 patients. They assessed whether ProGRP or NSE was elevated earlier than symptoms in radiographic findings and identified the correlation between the ProGRP or NSE levels at relapse and the response to salvage therapy retrospectively. They revealed that the serum NSE level at relapse was a good factor for predicting $\mathrm{CR}$ to salvage chemotherapy and a useful prognostic marker after relapse in patients with SCLC who achieved CR or PR to first-line chemotherapy. ${ }^{32}$ Their results are not in accordance with those of the present study. These conflicting results may be due to the differences 
in study design and grouping method. Liu et al reported that PLR and NLR were prognostic factors in SCLC and markers for inflammatory response. ${ }^{31}$ Miura et al also indicated that preoperative serum Alb level was important in predicting OS and recurrence-free survival in patients with postoperative non-SCLC. ${ }^{33} \mathrm{Zhu}$ et al showed that increased plasma D-Dimer levels in patients with cancer usually indicated an unfavorable prognosis. ${ }^{17}$ In the present study, no significant differences were observed among the above parameters, possibly because few studies placed these parameters together and compared them. When they were placed together, only LDH and Fbg were useful in predicting OS.

Furthermore, evaluation of the predictive accuracy revealed that combining $\mathrm{LDH}$ and Fbg was superior to $\mathrm{LDH}$ or Fbg alone, and it could significantly improve the AUC for the prediction of OS. Thus, the above results suggested that combining LDH and Fbg may be considered as one of the prognostic predictors among patients with SCLC in clinical practice. The statistically significant difference in AUC and the excellent specificity could enhance the reliability of OS prediction, which is crucial for making the pretreatment plan and the communication between doctors and patients.

This study had several limitations. First, it was a single-center, retrospective study with a relatively small sample size. A new cut-off value may be obtained when examining a large number of cases. Second, the type of treatment was not standardized across the included studies, where treatment ranged from sequential radiotherapy to synchronous radiotherapy. The time that a patient underwent radiation was usually chosen at the doctor's discretion; therefore, poor consistency existed across the study populations and even within cohorts. Third, no validation cohort was included to verify the findings. A prospective multicenter study with a considerably large dataset is needed to further validate the robustness and repeatability of the prediction model. Finally, the stage of SCLC varied among the studies. Patients with LD or ED stage were all included, which may have caused selection bias.

In conclusion, combined $\mathrm{LDH}$ and Fbg is an available and economic measurement in a standard clinical setting for SCLC. Therefore, using combined LDH and Fbg as predictive and prognostic markers for patients with SCLC is practical and straightforward. Future prospective cohort and multicenter studies are needed to find a proper cut-off value.

\section{Author Contributions}

All authors made substantial contributions to conception and design, acquisition of data, or analysis and interpretation of data; took part in drafting the article or revising it critically for important intellectual content; agreed to submit to the current journal; gave final approval of the version to be published; and agree to be accountable for all aspects of the work. Dr Wen Huang and Dr Ping Liu should be considered co-first authors.

\section{Funding}

This study was supported by the National Natural Science Foundation of China (No. 81870054) and the Key Project of National Science \& Technology for Infectious Diseases of China(No. 2018ZX10722301-002).

\section{Disclosure}

The authors report no conflicts of interest in this work.

\section{References}

1. Herbst RS, Heymach JV, Lippman SM. Lung cancer. $N$ Engl J Med. 2008;359(13):1367-1380. doi:10.1056/NEJMra0802714

2. Nilssen Y, Strand TE, Fjellbirkeland L, et al. Lung cancer survival in Norway, 1997-2011: from nihilism to optimism. Eur Respir J. 2016;47(1):275-287. doi:10.1183/13993003.00650-2015

3. Grivaux M, Duvert B, Ferrer-Lopez P, et al. One-year survival improvement in lung cancer in France. Results of the prospective real life studies KBP-2000-CPHG and KBP-2010-CPHG. Rev Pneumol Clin. 2016;72 (3):163-170. doi:10.1016/j.pneumo.2016.02.002

4. Li S, Yang Q, Wang H, et al. Prognostic significance of serum lactate dehydrogenase levels in Ewing's sarcoma: a meta-analysis. Mol Clin Oncol. 2016;5(6):832-838. doi:10.3892/mco.2016.1066

5. Ren X, Zhang Y, Lyu Y, et al. Lactate dehydrogenase and serum tumor markers for predicting metastatic status in geriatric patients with lung adenocarcinoma. Cancer Biomark. 2019;26(2):139-150. doi:10.3233/CBM-190201

6. Pelizzari G, Basile D, Zago S, et al. Lactate Dehydrogenase (LDH) response to first-line treatment predicts survival in metastatic breast cancer: first clues for a cost-effective and dynamic biomarker. Cancers (Basel). 2019;11(9):1243. doi:10.3390/cancers11091243

7. Tas F, Aykan F, Alici S, et al. Prognostic factors in pancreatic carcinoma: serum LDH levels predict survival in metastatic disease. Am J Clin Oncol. 2001;24(6):547-550. doi:10.1097/00000421200112000-00003

8. Hermes A, Waschki B, Reck M. Hyponatremia as prognostic factor in small cell lung cancer: a retrospective single institution analysis. Respir Med. 2012;106(6):900-904. doi:10.1016/j.rmed.2012.02.010

9. Petrelli F, Cabiddu M, Coinu A, et al. Prognostic role of lactate dehydrogenase in solid tumors: a systematic review and meta-analysis of 76 studies. Acta Oncol. 2015;54(7):961-970. doi:10.3109/0284186X.2015.1043026

10. Zhang X, Guo M, Fan J, et al. Prognostic significance of serum LDH in small cell lung cancer: a systematic review with meta-analysis. Cancer Biomark. 2016;16(3):415-423. doi:10.3233/CBM-160580

11. Mantovani A, Allavena P, Sica A, et al. Cancer-related inflammation. Nature. 2008;454(7203):436-444. doi:10.1038/nature07205

12. Go SI, Kim RB, Song HN, et al. Prognostic significance of the lymphocyte-to-monocyte ratio in patients with small cell lung cancer. Med Oncol. 2014;31(12):323. doi:10.1007/s12032-014-0323-y

13. Wang Z, Zhan P, Lv Y, et al. Prognostic role of pretreatment neutrophil-to-lymphocyte ratio in non-small cell lung cancer patients treated with systemic therapy: a meta-analysis. Transl Lung Cancer Res. 2019;8(3):214-226. doi:10.21037/tlcr.2019.06.10 
14. Diem S, Schmid S, Krapf M, et al. Neutrophil-to-Lymphocyte ratio (NLR) and Platelet-to-Lymphocyte ratio (PLR) as prognostic markers in patients with non-small cell lung cancer (NSCLC) treated with nivolumab. Lung Cancer. 2017;111:176-181. doi:10.1016/j. lungcan.2017.07.024

15. Ozmen S, Timur O, Calik I, et al. Neutrophil-lymphocyte ratio (NLR) and platelet-lymphocyte ratio (PLR) may be superior to C-reactive protein (CRP) for predicting the occurrence of differentiated thyroid cancer. Endocr Regul. 2017;51(3):131-136. doi:10.1515/enr-2017-0013

16. Fan S, Guan Y, Zhao G, et al. Association between plasma fibrinogen and survival in patients with small-cell lung carcinoma. Thorac Cancer. 2018;9(1):146-151. doi:10.1111/1759-7714.12556

17. Zhu LR, Li J, Chen P, et al. Clinical significance of plasma fibrinogen and D-dimer in predicting the chemotherapy efficacy and prognosis for small cell lung cancer patients. Clin Transl Oncol. 2016;18 (2):178-188. doi:10.1007/s12094-015-1350-7

18. Pichler M, Hutterer GC, Stojakovic T, et al. High plasma fibrinogen level represents an independent negative prognostic factor regarding cancer-specific, metastasis-free, as well as overall survival in a European cohort of non-metastatic renal cell carcinoma patients. Br J Cancer. 2013;109(5):1123-1129. doi:10.1038/bjc.2013.443

19. Takeuchi H, Ikeuchi S, Kitagawa Y, et al. Pretreatment plasma fibrinogen level correlates with tumor progression and metastasis in patients with squamous cell carcinoma of the esophagus. $J$ Gastroenterol Hepatol. 2007;22(12):2222-2227. doi:10.1111/ j.1440-1746.2006.04736.x

20. Monraats PS, Rana JS, Zwinderman AH, et al. -455G/A polymorphism and preprocedural plasma levels of Fibrinogen show no association with the risk of clinical restenosis in patients with coronary stent placement. Thromb Haemost. 2005;93(3):564-569. doi:10.1160/TH04-11-0708

21. Mosesson MW. Fibrinogen and fibrin structure and functions. J Thromb Haemost. 2005;3(8):1894-1904. doi:10.1111/j.15387836.2005.01365.x

22. Buccheri G, Ferrigno D, Ginardi C, et al. Haemostatic abnormalities in lung cancer: prognostic implications. Eur J Cancer. 1997;33 (1):50-55. doi:10.1016/S0959-8049(96)00310-3

23. Lillicrap D. Introduction to a series of reviews on cancer-associated thrombotic disease. Blood. 2013;122(10):1687-1688. doi:10.1182/ blood-2013-05-499087
24. Balkwill F, Mantovani A. Inflammation and cancer: back to Virchow? Lancet. 2001;357(9255):539-545. doi:10.1016/S0140-6736(00)04046-0

25. Palumbo JS, Kombrinck KW, Drew AF, et al. Fibrinogen is an important determinant of the metastatic potential of circulating tumor cells. Blood. 2000;96(10):3302-3309. doi:10.1182/blood.V96.10.3302

26. Palumbo JS, Potter JM, Kaplan LS, et al. Spontaneous hematogenous and lymphatic metastasis, but not primary tumor growth or angiogenesis, is diminished in fibrinogen-deficient mice. Cancer Res. 2002;62 (23):6966-6972.

27. Oh JR, Seo JH, Chong A, et al. Whole-body metabolic tumour volume of $18 \mathrm{~F}-\mathrm{FDG} \mathrm{PET} / \mathrm{CT}$ improves the prediction of prognosis in small cell lung cancer. Eur J Nucl Med Mol Imaging. 2012;39 (6):925-935. doi:10.1007/s00259-011-2059-7

28. Kuo YW, Jerng JS, Shih JY, et al. The prognostic value of the simplified comorbidity score in the treatment of small cell lung carcinoma. J Thorac Oncol. 2011;6(2):378-383. doi:10.1097/ JTO.0b013e3181fd4107

29. Harmsma M, Schutte B, Ramaekers FC. Serum markers in small cell lung cancer: opportunities for improvement. Biochim Biophys Acta. 2013;1836(2):255-272. doi:10.1016/j.bbcan.2013.06.002

30. Koukourakis MI, Giatromanolaki A, Sivridis E, et al. Lactate dehydrogenase-5 (LDH-5) overexpression in non-small-cell lung cancer tissues is linked to tumour hypoxia, angiogenic factor production and poor prognosis. Br J Cancer. 2003;89(5):877-885. doi:10.1038/ sj.bjc. 6601205

31. Liu D, Huang Y, Li L, et al. High neutrophil-to-lymphocyte ratios confer poor prognoses in patients with small cell lung cancer. $B M C$ Cancer. 2017;17(1):882. doi:10.1186/s12885-017-3893-1

32. Hirose T, Okuda K, Yamaoka T, et al. Are levels of pro-gastrinreleasing peptide or neuron-specific enolase \pm at relapse prognostic factors after relapse in patients with small-cell lung cancer? Lung Cancer. 2011;71(2):224-228. doi:10.1016/j.lungcan.2010.05.004

33. Miura K, Hamanaka K, Koizumi T, et al. Clinical significance of preoperative serum albumin level for prognosis in surgically resected patients with non-small cell lung cancer: comparative study of normal lung, emphysema, and pulmonary fibrosis. Lung Cancer. 2017;111:88-95. doi:10.1016/j.lungcan.2017.07.003

\section{Publish your work in this journal}

Cancer Management and Research is an international, peer-reviewed open access journal focusing on cancer research and the optimal use of preventative and integrated treatment interventions to achieve improved outcomes, enhanced survival and quality of life for the cancer patient.
The manuscript management system is completely online and includes a very quick and fair peer-review system, which is all easy to use. Visit http://www.dovepress.com/testimonials.php to read real quotes from published authors. 